\title{
A Class of Optimal Liquidation Problem with a Nonlinear Temporary Market Impact
}

\author{
Jiangming $\mathrm{Ma} \mathbb{D}^{1}$ and Di Gao $\mathbb{D}^{2}$ \\ ${ }^{1}$ School of Economics, Xihua University, Chengdu, Sichuan 610039, China \\ ${ }^{2}$ School of International Business, Southwestern University of Finance and Economics, Chengdu, Sichuan 611130, China \\ Correspondence should be addressed to Di Gao; gaodi1593@163.com
}

Received 14 October 2020; Revised 29 November 2020; Accepted 11 December 2020; Published 24 December 2020

Academic Editor: Mohamed El Ghami

Copyright (c) 2020 Jiangming Ma and Di Gao. This is an open access article distributed under the Creative Commons Attribution License, which permits unrestricted use, distribution, and reproduction in any medium, provided the original work is properly cited.

\begin{abstract}
We extend the self-exciting model by assuming that the temporary market impact is nonlinear and the coefficient of the temporary market impact is an exponential function. Through optimal control method, the optimal strategy satisfies the second-order nonlinear ordinary differential equation. The specific form of the optimal strategy is given, and the decreasing property of the optimal strategy is proved. A numerical example is given to illustrate the financial implications of the model parameter changes. We find that the optimal strategy of a risk-neutral investor changes with time and investment environment.
\end{abstract}

\section{Introduction}

In the financial field, the problem of optimal liquidation is widely studied. In 1998, Bertsimas and Lo [1] study the minimum transaction completion in the case of fixed trading time dynamic trading strategy. Based on the original scholar's model, Almgren and Chriss [2] consider the expected costs and risks of execution and propose a simple market impact model. It includes the following three parts: unaffect price process, temporary market impact, and permanent. Almgren-Chriss market impact model provides a good tool to continue studying the optimal liquidation problem. Almgren [3] gives the optimal execution strategy under the nonlinear temporary impact. Curato et al. [4] study the optimal execution of a large trade when the transient impact is nonlinear. Gueant and Lehalle [5] carry out research on optimal liquidation when the execution process intensity is general functional forms.

Some scholars research the corresponding optimal liquidation strategy under the expanded Almgren-Chriss model. Schied and Gatheral $[6,7]$ show the optimal strategies when the unaffected price process is geometric Brownian motion. Lehalle and Neuman [8] obtain the optimal strategies and provide the existence and uniqueness of them when the model incorporates a Markovian signal. When order flow is imbalanced and uncertain, the optimal execution is discussed by Bechler and Ludkovski [9] and Cheng et al. [10], respectively. Cartea and Jaimungal [11] and Gueant et al. [12] address the optimal liquidation when the order book is limited. Many scholars continue to make further research studies in the recent years. Cartea and Jaimungal [13] investigate optimal execution when the investor executes a large order. Kato [14] gets the optimal execution of trader when the volume weighted average price (VWAP) is used in the Almgren-Chriss model. Frei and Westray [15] propose a relative volume curve model under the VWAP model and get the explicit characterization of optimal execution. Based on [14], Kato [16] obtains a second-order asymptotic expansion formula of optimal strategies by the penalization method. Klöck et al. [17] change the application scenario and study the execution with dark pool in the Almgren-Chriss model. Bela et al. [18] study the optimal liquidation under the Almgren-Chriss model with running and terminal inventory costs and general predictive signals about price changes. Bank et al. [19] carry out research on the optimal problem of hedging and give the general predictable target hedging strategies. In addition, some scholars investigate the optimal liquidation by using 
new methods. Damian [20] discusses the optimal execution under the multitime version of the Almgren-Chriss model by the variational calculus techniques which assumes that the optimal control is in the set of admissible controls. Bismuth et al. [21] address the optimal liquidation in an Almgren-Chriss framework by the Bayesian learning and dynamic programming techniques when expected returns are unknown. Besides, Schied and Zhang [22] consider the Almgren-Chriss model has $n$ risk-averse agents and prove the property of optimal liquidation strategies.

Differential equations are widely used in engineering. Wakif et al. $[23,24]$ study the stability of nanofluids which has the characteristics of electrically conducting and Newtonian fluids, incorporating the effects of thermophoresis and Brownian motion in different situations. Then, they get the corresponding differential equations which are obtained by the relevant methods. From the numerical methods, they discuss the properties and get the solutions of differential equations. Similarly, differential equations are also used in the financial field. The optimal strategies of relevant literature mentioned above satisfy the differential equations through the optimal control methods.

Caye and Muhle-Karbe [25] consider that the trades not only incur price impact but also increase the execution costs. Thus, they propose a self-exciting price model and get the optimal liquidation strategies under the Almgren-Chriss framework. However, they only discuss the temporary impact, and its coefficients are linear functions. Different from the above references, we suppose that the temporary impact and its coefficients are nonlinear functions. Namely, let the temporary impact and its coefficient be the exponential function and the power function, respectively, which are used in economic and finance research. Finally, we get the specific form and prove the properties of optimal liquidation.

The paper is organized as follows. In Section 2, we state the Almgren-Chriss framework, self-exciting price model, and objective function. In Section 3, we give the specific form of optimal liquidation and discuss the properties of solutions. In Section 4, we show the numerical examples and the corresponding financial interpretations.

\section{Statement of Background}

In this paper, we use the continuous-time market impact model of Almgren-Chriss which supposes that the active time of every investor is fixed in $[0, T]$. An investor hold $x$ shares at the initial time and completely trade at the time $T$, that is, $X_{0}=x$ and $X_{T}=0$. The investor's strategy is $X_{t}$ which is absolutely continuous and bounded with derivative $\dot{X}_{t}$ and $X_{t}=x+\int_{0}^{t} \dot{X}_{t} \mathrm{~d} t$, where $\dot{X}_{t}$ satisfies $\int_{0}^{T}\left(\dot{X}_{t}\right)^{2}<\infty$.

A filtration $\left(\mathscr{F}_{t}\right)_{t>0}$ on the given probability space $(\Omega, \mathscr{F}, p)$ is supported by the standard Brownian motion $W_{t}$. We suppose that the a risk asset's unaffected price process follows the Bachelier [26] model with no drift:

$$
S_{t}^{0}:=\sigma d W_{t} .
$$

The Almgren-Chriss model is supposed that the price of a risk asset is related to the hold share and trading speed at the time $t$. So, the Almgren-Chriss market impact model is divided into three components: unaffected price process, permanent impact components, and temporary impact components. The specific form of the Almgren-Chriss model is assumed to be

$$
S_{t}:=S_{t}^{0}+\gamma\left(X_{t}-x\right)+\lambda \dot{X}_{t},
$$

where $\gamma\left(X_{t}-x\right)$ and $\dot{X}_{t}$ represents the permanent impact and temporary impact components, respectively; the parameters $\lambda>0$ and $\gamma>0$ represent the coefficient of permanent and temporary impact components.

Cayé and Muhle-Karbe [25] give the self-exciting price impact under the Almgren-Chriss framework. In this model, the parameter of temporary impact component is a linear function about the number of shares already sold. The specific form is assumed to be

$$
S_{t}:=S_{t}^{0}+\left(a+b\left(x-X_{t}\right)\right) \dot{X}_{t}, \quad t \in[0, T],
$$

where $a>0$ and $b>0$. In equation (3), there is no permanent impact component because the influence of the permanent impact component about the cost of investor is fixed.

At each time $t \in[0, T]$, the infinitesimal amounts of $-\dot{X}_{t} \mathrm{~d} t$ shares are sold at price $S_{t}$. Therefore, the total implementation cost is represented by

$$
C(X):=x S_{0}+\int_{0}^{T} \dot{X}_{t} S_{t} \mathrm{~d} t .
$$

So, the optimal trade execution problem becomes the minimization of expected costs. We only need to solve the minimization of expected cost:

$$
\operatorname{minimize} \mathbb{E}[C(X)] \text {. }
$$

Problem (5) is proposed by Bertsimas and Lo [1]. Carmona and Yang [27] use (5) to deal with the problem of the maximization.

\section{Main Results}

Cayé and Muhle-Karbe [25] only discuss the coefficient of temporary impact component is a linear function. However, in the real lifetime, the coefficient of temporary impact component maybe nonlinear. So, we suppose that the coefficient of temporary impact component like the exponential function is widely used in economic activities. Thus, the coefficient of temporary impact component is assumed to be $e^{a+b\left(x-X_{t}\right)}$, where $a>0$ and $b>0$.

Theorem 1. Since the coefficient of temporary impact component is $e^{a+b\left(x-X_{t}\right)}$, there exists a unique strategy for mean optimization. The strategy is the unique solution of the following differential equation:

$$
\ddot{X}_{t}-\frac{b}{2} \dot{X}_{t}^{2}=0,
$$

with two-point boundary conditions 


$$
\begin{aligned}
& X_{0}=x, \\
& X_{T}=0 .
\end{aligned}
$$

The solution of equation (6) is

$$
t=C_{1}+C_{2} \int e^{-(b / 2) X_{t}} \mathrm{~d} X_{t}
$$

Proof. When the coefficient of temporary impact component is $e^{a+b\left(x-X_{t}\right)}$, equation (3) is

$$
S_{t}:=S_{t}^{0}+e^{a+b\left(x-X_{t}\right)} \dot{X}_{t}, \quad t \in[0, T] .
$$

From equations (4) and (9), we obtain

$$
\begin{aligned}
C(X) & :=x S_{0}+\int_{0}^{T} \dot{X}_{t} S_{t} \mathrm{~d} t \\
& =x S_{0}+\int_{0}^{T} \dot{X}_{t}\left(S_{t}^{0}+\left(e^{a+b\left(x-X_{t}\right)} \dot{X}_{t}\right) \mathrm{d} t\right) \\
& =\int_{0}^{T} \sigma X_{t} d W_{t}+\int_{0}^{T} e^{a+b\left(x-X_{t}\right)} \dot{X}_{t}^{2} \mathrm{~d} t .
\end{aligned}
$$
obtain

From the properties of Ito integral and equation (5), we

$$
\operatorname{minimize} \mathbb{E}[C(X)]=\int_{0}^{T} e^{a+b\left(x-X_{t}\right)} \dot{X}_{t}^{2} \mathrm{~d} t .
$$

In order to get the solution of equation (11), we use the Euler-Lagrange equation to get the second-order ordinary differential equation:

$$
\ddot{X}_{t}-\frac{b}{2} \dot{X}_{t}^{2}=0
$$

The optimal strategy satisfies equation (12). From [28], the solution of equation (12) is

$$
t=C_{1}+C_{2} \int e^{-(b / 2) X_{t}} \mathrm{~d} X_{t}
$$

Theorem 2. The optimal strategy from equations (12) and (11), over all deterministic, absolutely continuous strategies $X_{t}$, is decreasing.

$$
\begin{aligned}
& \text { Proof } \\
& \qquad C(X)=\int_{0}^{T} e^{a+b\left(x-X_{t}\right)} \dot{X}_{t}{ }^{2} \mathrm{~d} t=e^{a+b x} \int_{0}^{T} e^{-X_{t}} \dot{X}_{t}{ }^{2} \mathrm{~d} t=\mathrm{F}(X) .
\end{aligned}
$$

Let $Y=X-X^{*}$; then, we obtain

$$
\begin{aligned}
& \mathscr{F}(X)=\mathscr{F}\left(Y+X^{*}\right) \\
& =e^{a+b x} \int_{0}^{T} e^{-X_{t}^{*}-Y_{t}}\left(\dot{X}_{t}^{*}+\dot{Y}_{t}\right)^{2} \mathrm{~d} t \\
& =e^{a+b x} \int_{0}^{T} e^{-X_{t}^{*}-Y_{t}}\left(\dot{X}_{t}^{*}{ }^{2}+2 \dot{X}_{t}^{*} \dot{Y}_{t}+\dot{Y}_{t}^{2}\right) \mathrm{d} t \\
& =e^{a+b x} \int_{0}^{T} e^{-X_{t}^{*}-Y_{t}} \dot{X}_{t}^{*} \mathrm{~d} t+e^{a+b x} \int_{0}^{T} e^{-X_{t}^{*}-Y_{t}} 2 \dot{X}_{t}^{*} \dot{Y}_{t} \mathrm{~d} t \\
& +e^{a+b x} \int_{0}^{T} e^{-X_{t}^{*}-Y_{t}} \dot{Y}_{t}{ }^{2} \mathrm{~d} t \\
& \geq e^{a+b x} \int_{0}^{T} e^{-X_{t}^{*}-Y_{t}} \dot{X}_{t}^{*}{ }^{2} \mathrm{~d} t \\
& \geq e^{a+b x} \int_{0}^{T} e^{-X_{t}^{*}} \dot{X}_{t}^{*}{ }^{2} \mathrm{~d} t .
\end{aligned}
$$

Because the Almgren-Chriss model supposes that there is no existence price manipulation, the $\dot{X}_{t}$ satisfies $\dot{X}_{t}<0$. Thus, $X_{t}$ is decreasing. From the properties of exponential function and integral, we get the proof of Theorem 2 .

Except for references [3, 5, 10], there are still some scholars studying the problem of optimal execution when temporary impact is nonlinear. Gatheral [29] discusses the optimal liquidation problems under the basic assumption of the Almgren-Chriss model which contains some special nonlinear temporary market impact function. When the temporary market impact function in the Almgren-Chriss model is nonlinear, Labadie and Lehalle [30] examine the optimal starting times, stopping times, and risk measures for algorithmic trading of target close and implementation shortfall. Hendricks and Wilcox [31] research the optimal trade execution of the Almgren-Chriss framework by a reinforcement learning method. Horst and Naujokat [32] show the value derivatives under market impact in a multiplayer framework which is based on the nonlinear temporary market impact function of the Almgren-Chriss model.

Although Caye and Muhle-Karbe [25] pay attention to the optimal liquidation of self-exciting price impact under the Almgren-Chriss framework, the case of nonlinear temporary market impact function is not studied. Next, we suppose that the temporary market impact function likes a nonlinear form $h\left(X_{t}\right)$. Since $h\left(\dot{X}_{t}\right)$ has many forms, drawing on the above research studies of optimal liquidation with the nonlinear functions, we let $h\left(X_{t}\right)$ be the power function. Namely, $h\left(\dot{X}_{t}\right)$ has the form $h\left(\dot{X}_{t}\right)=\left(\dot{X}_{t}\right)^{\alpha}, \alpha>0$. However, in the actual process of 
solution, it is difficult to get the general solution of optimizing equation when the $h\left(\dot{X}_{t}\right)$ is the power function. Therefore, we research the special case which is usually used in economic and finance and discuss the optimal strategies when $h\left(\dot{X}_{t}\right)=\left(\dot{X}_{t}\right)^{2}$. Thus, equation (3) is changed for

$$
S_{t}:=S_{t}^{0}+\left(a+b\left(x-X_{t}\right)\right)\left(\dot{X}_{t}\right)^{2}, \quad t \in[0, T] .
$$

Theorem 3. Since the temporary impact component is $h\left(\dot{X}_{t}\right)=\left(\dot{X}_{t}\right)^{2}$, there exists a unique strategy for mean optimization. The strategy is the unique solution of the following differential equation:

$$
3\left(a+b\left(x-X_{t}\right)\right) \ddot{X}_{t}-b \dot{X}_{t}^{2}=0,
$$

with two-point boundary conditions

$$
\begin{aligned}
& X_{0}=x, \\
& X_{T}=0 .
\end{aligned}
$$

The solution of equation (17) is

$$
\left(a+b\left(x-X_{t}\right)\right)^{(2 / 3)}=C_{1} t+C_{2} .
$$

Proof. When the coefficient of temporary impact component is $e^{a+b\left(x-X_{t}\right)}$, equation ( 3 ) is

$$
S_{t}:=S_{t}^{0}+\left(a+b\left(x-X_{t}\right)\right)\left(\dot{X}_{t}\right)^{2}, \quad t \in[0, T] .
$$

From equations (4) and (20), we obtain

$$
\begin{aligned}
C(X) & :=x S_{0}+\int_{0}^{T} \dot{X}_{t} S_{t} \mathrm{~d} t \\
& =x S_{0}+\int_{0}^{T} \dot{X}_{t}\left(S_{t}^{0}+\left(a+b\left(x-X_{t}\right)\right)\left(\dot{X}_{t}\right)^{2}\right) \mathrm{d} t \\
& =\int_{0}^{T} \sigma X_{t} \mathrm{~d} W_{t}+\int_{0}^{T}\left(a+b\left(x-X_{t}\right)\right) \dot{X}_{t}^{3} \mathrm{~d} t .
\end{aligned}
$$
obtain

By the properties of Ito integral and equation (5), we

$$
\operatorname{minimize} \mathbb{E}[C(X)]=\int_{0}^{T}\left(a+b\left(x-X_{t}\right)\right) \dot{X}_{t}^{3} \mathrm{~d} t .
$$

In order to get the solution of equation (22), we use the Euler-Lagrange equation to get the second-order ordinary differential equation. The optimal strategy satisfies the following equation:

$$
3\left(a+b\left(x-X_{t}\right)\right) \ddot{X}_{t}-b \dot{X}_{t}^{2}=0 .
$$

From [28], the solution of equation (23) is

$$
\left(a+b\left(x-X_{t}\right)\right)^{(2 / 3)}=C_{1} t+C_{2} .
$$

Theorem 4. The optimal strategy from equations (17) and (24), over all deterministic, absolutely continuous strategies $X_{t}$, is decreasing.
Proof

$$
\begin{aligned}
C(X) & =\int_{0}^{T}\left(a+b\left(x-X_{t}\right)\right) \dot{X}_{t}{ }^{3} \\
\mathrm{~d} t & =(a+b x) \int_{0}^{T}\left(-b X_{t}\right) \dot{X}_{t}{ }^{3} \mathrm{~d} t=\mathscr{F}(X) .
\end{aligned}
$$

Let $Y=X-X^{*}$; then, we obtain

$$
\begin{aligned}
\mathscr{F}(X)= & \mathscr{F}\left(Y+X^{*}\right)=(a+b x) \int_{0}^{T}\left(-b X_{t}^{*}-b Y_{t}\right)\left(\dot{X}_{t}^{*}+\dot{Y}_{t}\right)^{3} \mathrm{~d} t \\
= & (a+b x) \int_{0}^{T}\left(-b X_{t}^{*}-b Y_{t}\right)\left(\dot{X}_{t}^{*}+3 \dot{X}_{t}^{*} \dot{Y}_{t}\right. \\
& \left.+3 \dot{X}_{t}^{*} \dot{Y}_{t}^{2}+\dot{Y}_{t}^{3}\right) \mathrm{d} t \\
= & (a+b x) \int_{0}^{T}\left(-b X_{t}^{*}-b Y_{t}\right) \dot{X}_{t}^{*} \mathrm{~d} t \\
& +(a+b x) \int_{0}^{T}\left(-b X_{t}^{*}-b Y_{t}\right)\left(3 \dot{X}_{t}^{*} \dot{Y}_{t}\right) \mathrm{d} t \\
& +(a+b x) \int_{0}^{T}\left(-b X_{t}^{*}-b Y_{t}\right)\left(3 \dot{X}_{t}^{*} \dot{Y}_{t}{ }^{2}\right) \mathrm{d} t \\
& +(a+b x) \int_{0}^{T}\left(-b X_{t}^{*}-b Y_{t}\right)\left(\dot{Y}_{t}^{3}\right) \mathrm{d} t \\
\geq & (a+b x) \int_{0}^{T}\left(-b X_{t}^{*}-b Y_{t}\right) \dot{X}_{t}^{*} \mathrm{~d} t \\
\geq & (a+b x) \int_{0}^{T}\left(-b X_{t}^{*} \dot{X}_{t}^{*}{ }^{3}\right) \mathrm{d} t .
\end{aligned}
$$

From the properties of $\dot{X}_{t}, \dot{X}_{t}<0$, and integral, we get the proof of Theorem 4 .

Next, we discuss the optimal liquidation strategies when the temporary impact function is power function and the coefficient of temporary impact is $e^{a+b\left(x-X_{t}\right)}$. Thus, the price process is changed to be

$$
S_{t}:=S_{t}^{0}+e^{a+b\left(x-X_{t}\right)}\left(\dot{X}_{t}\right)^{2}, \quad t \in[0, T] .
$$

Theorem 5. Since the temporary impact component is $h\left(\dot{X}_{t}\right)=\left(\dot{X}_{t}\right)^{2}$ and the coefficient of temporary impact component is $e^{a+b\left(x-X_{t}\right)}$, there exists a unique strategy for mean optimization. The strategy is the unique solution of the following differential equation:

$$
3 \ddot{X}_{t}-b \dot{X}_{t}^{2}=0
$$

with two-point boundary conditions

$$
\begin{aligned}
& X_{0}=x, \\
& X_{T}=0 .
\end{aligned}
$$

The solution of equation (6) is

$$
\left(a+b\left(x-X_{t}\right)\right)^{(2 / 3)}=C_{1} t+C_{2} .
$$

Proof. From equations (4) and (19), we obtain 


$$
\begin{aligned}
C(X) & :=x S_{0}+\int_{0}^{T} \dot{X}_{t} S_{t} \mathrm{~d} t, \\
& =x S_{0}+\int_{0}^{T} e^{a+b\left(x-X_{t}\right)}\left(\dot{X}_{t}\right)^{3} \mathrm{~d} t \\
& =\int_{0}^{T} \sigma X_{t} \mathrm{~d} W_{t}+\int_{0}^{T} e^{a+b\left(x-X_{t}\right)} \dot{X}_{t}{ }^{3} \mathrm{~d} t .
\end{aligned}
$$

Through the properties of Ito integral and equation (5), we have

$$
\operatorname{minimize} \mathbb{E}[C(X)]=\int_{0}^{T} e^{a+b\left(x-X_{t}\right)} \dot{X}_{t}^{3} \mathrm{~d} t .
$$

In order to get the solution of equation (32), we use the Euler-Lagrange equation to get the second-order ordinary differential equation:

$$
3 \ddot{X}_{t}-b \dot{X}_{t}^{2}=0 .
$$

The optimal strategy satisfies equation (33). From [28], the solution of equation (33) is

$$
t=C_{1}+C_{2} \int e^{-(b / 3) X_{t}} \mathrm{~d} X_{t} .
$$

Theorem 6. The optimal strategy from equations (28) and (34), over all deterministic, decreasing, and absolutely continuous strategies $x$ with square-integrable derivative, satisfies $X_{0}=x$ and $X_{T}=0$.

\section{Proof}

$$
\begin{aligned}
C(X) & =\int_{0}^{T} e^{a+b\left(x-X_{t}\right)} \dot{X}_{t}{ }^{3} \mathrm{~d} t \\
& =(a+b x) \int_{0}^{T}\left(-b X_{t}\right) \dot{X}_{t}^{3} \mathrm{~d} t=\mathscr{F}(X) .
\end{aligned}
$$

Let $Y=X-X^{*}$; then, we obtain

$$
\begin{aligned}
\mathscr{F}(X)=\mathscr{F}\left(Y+X^{*}\right)= & e^{(a+b x)} \int_{0}^{T} e^{\left(-b X_{t}^{*}-b Y_{t}\right)}\left(\dot{X}_{t}^{*}+\dot{Y}_{t}\right)^{3} \mathrm{~d} t \\
= & e^{(a+b x)} \int_{0}^{T} e^{\left(-b X_{t}^{*}-b Y_{t}\right)}\left(\dot{X}_{t}^{*}{ }^{3}+3 \dot{X}_{t}^{*} \dot{Y}_{t}\right. \\
& \left.+3 \dot{X}_{t}^{*} \dot{Y}_{t}{ }^{2}+\dot{Y}_{t}{ }^{3}\right) \mathrm{d} t \\
= & e^{(a+b x)} \int_{0}^{T} e^{\left(-b X_{t}^{*}-b Y_{t}\right)} \dot{X}_{t}^{*} \mathrm{~d} t \\
& +(a+b x) \int_{0}^{T} e^{\left(-b X_{t}^{*}-b Y_{t}\right)}\left(3 \dot{X}_{t}^{*} \dot{Y}_{t}\right) \mathrm{d} t \\
& +e^{(a+b x)} \int_{0}^{T} e^{\left(-b X_{t}^{*}-b Y_{t}\right)}\left(3 \dot{X}_{t}^{*} \dot{Y}_{t}{ }^{2}\right) \mathrm{d} t \\
& +e^{(a+b x)} \int_{0}^{T} e^{\left(-b X_{t}^{*}-b Y_{t}\right)}\left(\dot{Y}_{t}{ }^{3}\right) \mathrm{d} t \\
\geq & e^{(a+b x)} \int_{0}^{T} e^{\left(-b X_{t}^{*}-b Y_{t}\right)} \dot{X}_{t}^{*} \mathrm{~d} t \\
\geq & e^{(a+b x)} \int_{0}^{T} e^{-b X_{t}^{*} \dot{X}_{t}^{*}} \mathrm{~d} t .
\end{aligned}
$$

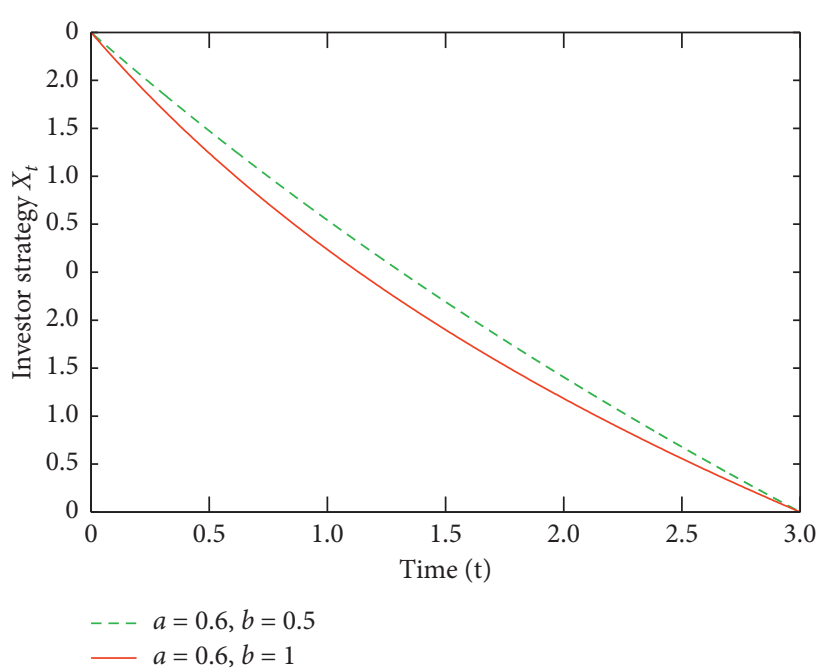

FIgURE 1: Equation (13).

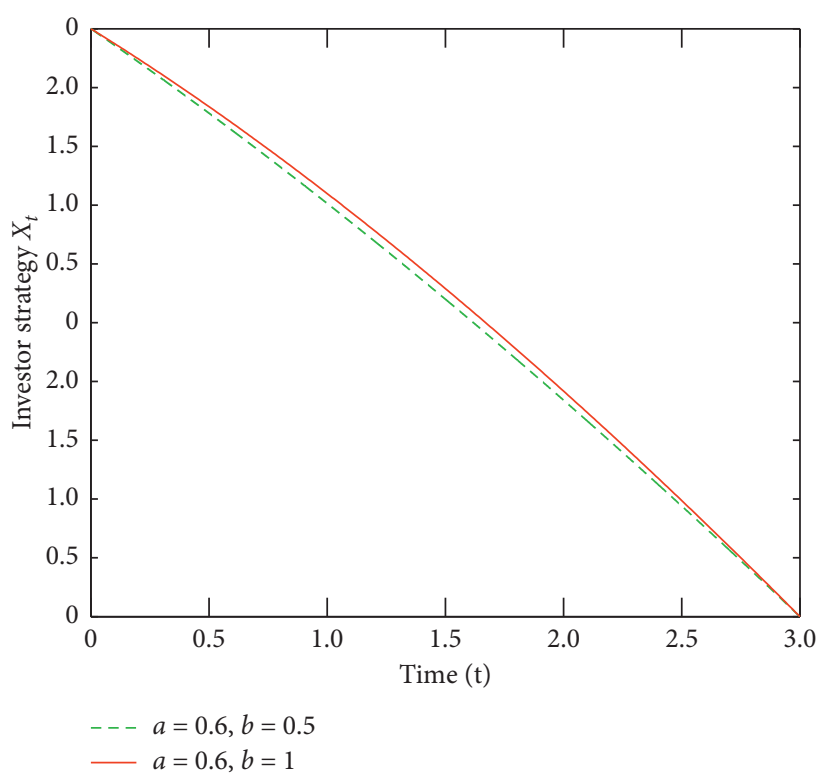

Figure 2: Equation (24).

From the properties of $\dot{X}_{t}, \dot{X}_{t}<0$, and integral, we get the proof of Theorem 6 .

\section{Numerical Simulation}

In the previous part, we give the specific forms of optimal investment strategies for risk-neutral investors when the temporary market impact and coefficient of temporary market impact are a power function and an exponential function, respectively. According to the parameter setting method in the relevant literature, we assume that $X_{0}=2$, $X_{T=3}=0$, and $t \in[0,3]$, and the values of other parameters are shown in the figures.

From equation (13), we know that the optimal liquidation has nothing to do with $a$. From Figure 1, we get that the cost of trading becomes higher when $b$ gets larger. Thus, 


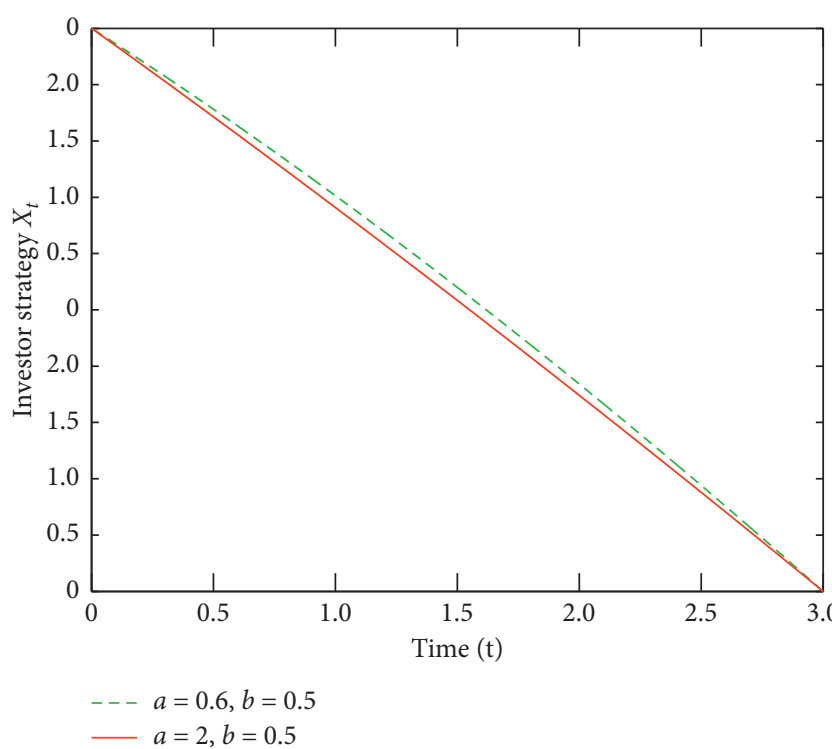

Figure 3: Equation (24).

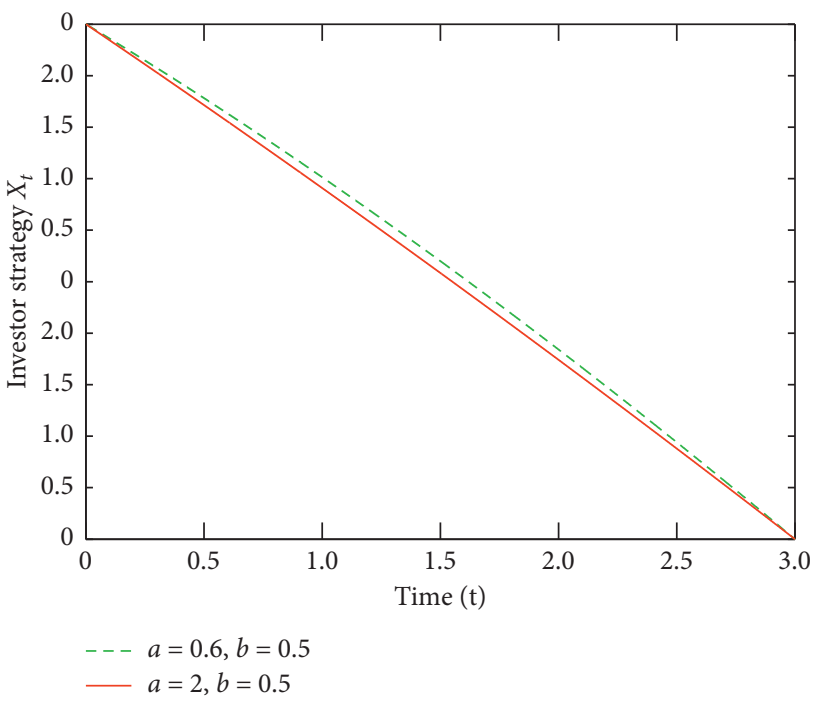

Figure 4: Equation (34).

the investor speeds up liquidation early. From equation (24) and Figures 2 and 3, when the temporary market impact is a power function and the coefficient of temporary market impact is a linear function, the investor realizes that they will face large execution costs with bigger $a$ and smaller $b$ so that they speed up liquidation early and slow down the trading speed later. When temporary market impact is a power function and the coefficient of temporary market impact is an exponential function, we find that $a$ has no effect on the optimal liquidation. In Figure 4, the bigger $b$ leads to increased costs of execution. Therefore, in order to decrease the costs, the investor will speed up liquidation early.

Through numerical examples, we find that when investment conditions change, the optimal investment strategy of risk-neutral investors is not the average of initial holdings with respect to time. However, it changes with time and investment environment. Since investment environment is complicated and volatile, the purpose of this paper is to remind risk-neutral investors that when they face the three investment environments, and they should follow these investment strategies to get the maximum return.

\section{Conclusion}

In this paper, combining the model setting of Cay and Muhle-Karbe [25] with the review of relevant literature, we put forward a class of optimal liquidation when the temporary market impact is a power function and the coefficient of temporary market impact is an exponential function, respectively. The optimal liquidation strategies satisfy the second-order nonlinear ordinary differential equations. The form of optimal liquidation strategies is given. At the same time, we discuss the properties of optimal liquidation strategies. Through the numerical example, we explain the financial implications with the changed parameter. This paper studies the optimal liquidation strategy of investors under three situations. In the future, more situations with financial implications will be discussed, particularly the fractional form of derivation with financial implications.

\section{Data Availability}

The data used to support the findings of this study are included within the article.

\section{Conflicts of Interest}

The authors declare that there are no conflicts of interest regarding the publication of this paper.

\section{Acknowledgments}

This work was supported by the National Natural Science Foundation of China (Grant 61903064), Key Research Base of Philosophy and Social Sciences for Colleges and Universities in Sichuan Province (KJJR2019-004), and Talent Introduction Project of Xihua University (w202247).

\section{References}

[1] D. Bertsmas and A. Lo, "Optimal execution of execution costs," Journal of Finance Markets, vol. 1, pp. 1-50, 1998.

[2] R. Almgren and N. Chriss, "Optimal execution of portfolio transactions," Journal of Risk, vol. 3, no. 2, pp. 5-39, 2000.

[3] R. F. Almgren, "Optimal execution with nonlinear impact functions and trading-enhanced risk," Applied Mathematical Finance, vol. 10, no. 1, pp. 1-18, 2003.

[4] G. Curato, J. Gatheral, and F. Lillo, "Optimal execution with non-linear transient market impact," Quantitative Finance, vol. 17, no. 1, pp. 41-54, 2017.

[5] O. Gueant and C. A. Lehalle, "General intersity shapes in optimal liquidation," Mathematical Finance, vol. 25, no. 3, pp. 457-495, 2015.

[6] A. Schied, "Robust strategies for optimal order execution in the almgren-chriss framework," Applied Mathematical Finance, vol. 20, no. 3, pp. 264-286, 2013. 
[7] J. Gatheral and A. Schied, "Optimal trade execution under geometric brownian motion in the almgren and Chriss framework," International Journal of Theoretical and Applied Finance, vol. 14, no. 3, pp. 353-368, 2011.

[8] C.-A. Lehalle and E. Neuman, "Incorporating signals into optimal trading," Finance and Stochastics, vol. 23, no. 2, pp. 275-311, 2019.

[9] K. Bechler and M. Ludkovski, "Optimal execution with dynamic order flow imbalance," SIAM Journal on Financial Mathematics, vol. 6, no. 1, pp. 1123-1151, 2015.

[10] X. Cheng, M. D. Giacinto, and T. Wang, "Optimal execution with uncertain order fills in Almgren-Chriss framework," Quantitative Finance, vol. 17, no. 1, pp. 55-69, 2017.

[11] A. Cartea and S. Jaimungal, "Optimal execution with limit and market orders," Quantitative Finance, vol. 15, no. 8, pp. 1279-1291, 2015.

[12] O. Gueant, C.-A. Lehalle, and J. Fernandez-Tapia, "Optimal portfolio liquidation with limit orders," SIAM Journal on Financial Mathematics, vol. 3, no. 1, pp. 740-764, 2012.

[13] A. Cartea and S. Jaimungal"Incorporating order-flow into optimal execution," Mathematics and Financial Economics, vol. 10, no. 3, pp. 339-364, 2016.

[14] T. Kato, "VWAP execution as an optimal strategy," JSIAM Letters, vol. 7, pp. 33-36, 2015.

[15] C. Frei and N. Westray, "Optimal Execution of A VWAP order: a stochastic control approach," Mathematical Finance, vol. 25, no. 3, pp. 612-639, 2015.

[16] T. Kato, "An optimal execution problem in the volume-dependent Almgren-Chriss model," Algorithmic Finance, vol. 17, pp. 1-14, 2018.

[17] F. Klöck, A. Schied, and Y. Sun, "Price manipulation in a market impact model with dark pool," Applied Mathematical Finance, vol. 24, no. 5, pp. 417-450, 2017.

[18] C. Bela, J. Muhle-Karbe, and K. Ou, "Liquidation in target zone models," Market Microstructure and Liquidity, vol. 4, no. 3, pp. 1-12, 2018.

[19] P. Bank, H. M. Soner, and M Vo, "Hedging with temporary price impact," Mathematics and Financial Economics, vol. 11, no. 2, pp. 215-239, 2017.

[20] V. Voß, "Modelling optimal execution strategies for Algorithmic trading," Theoretical and Applied Economics, vol. XXII, no. 4, pp. 99-104, 2015.

[21] A. Bismuth, O. Gueant, and J. Pu, "Portfolio choice, portfolio liquidation, and portfolio transition under drift uncertainty," Mathematics and Financial Economics, vol. 13, no. 4, pp. 661-719, 2019.

[22] A. Schied and T. Zhang, "A state-constrained differential game arising in optimal portfolio liquidation," Mathematical Finance, vol. 27, no. 3, pp. 779-802, 2017.

[23] A. Wakif, Z. Boulahia, F. Ali, M. R. Eid, and R. Sehaqui, "Numerical analysis of the unsteady natural convection MHD Couette nanofluid flow in the presence of thermal radiation using single and two-phase nanofluid models for CuCWater nanofluids," International Journal of Applied and Computational Mathematics, vol. 4, no. 81, pp. 1-27, 2018.

[24] A. Wakif, A. Chamkha, T. Thumma, I. L. Animasaun, and R. Sehaqui, "Thermal radiation and surface roughness efects on the thermo-magneto-hydrodynamic stability of aluminacopper oxide hybrid nanofuids utilizing the generalized Buongiorno's nanofuid model," Journal of Thermal Analysis and Calorimetry, 2020.

[25] T. Cayé and J. Muhle-Karbe, "Liquidation with self-exciting price impact," Mathmatical Finance Economic, vol. 10, no. 17, pp. 15-28, 2016.
[26] L. Bachelier, “Théorie de la spéculation," Annales scientifiques de l'École normale supérieure, vol. 17, no. 17, pp. 21-86, 1900.

[27] R. A. Carmona and Z. Joseph Yang, "Optimal liquidation under stochastic price impact," International Journal of Theoretical and Applied Finance, vol. 21, no. 8, pp. 1-28, 2018.

[28] D. P. Andrei and F. Z. Valentin, Handbook of Exact Solutions for Ordinary Differential Equations, Chapman Hall/CRC, Boca Raton, FL, USA, 2003.

[29] J. Gatheral, "No-dynamic-arbitrage and market impact," Quantitative Finance, vol. 10, no. 7, pp. 749-759, 2010.

[30] M. Labadie and C. Lehalle, "Optimal starting times, stopping times and risk measures for algorithmic trading: target close and implementation shortfall," 2013, https://arxiv.org/pdf/ 1205.3482.pdf.

[31] D. Hendricks and D. Wilcox, "A reinforcement learning extension to thealmgren-chriss framework for optimal trade execution," 2014, https://arxiv.org/pdf/1403.2229.pdf.

[32] U. Horst and F. Naujokat, "Illiquidity and derivative valuation," 2018, https://arxiv.org/pdf/0901.0091.pdf. 